\title{
ASOCIACIONES CONCEPTUALES, PRÁCTICAS, CRÍTICAS Y DE TENDENCIA EN LA CO-CREACIÓN DE VALOR: UNA REVISIÓN SISTEMÁTICA DE LITERATURA (RSL)
}

\author{
CONCEPTUAL, PRACTICES, CRITICAL AND CO-TRENDS ASSOCIATIONS \\ IN VALUE CO-CREATION: A SYSTEMATIC LITERATURE REVIEW (SLR)
}

Olga Lucía Hurtado-Cardona *1

1 Fundación Universitaria Comfamiliar Risaralda, Universidad Nacional de Colombia- Sede Manizales, Colombia. E-mail: ohurtado@uc.edu.co

El propósito de este trabajo es realizar una revisión sistemática de los documentos que caracterizan el uso de análisis estadísticos en torno a la co-creación de valor con el fin de encontrar puntos comunes y divergentes, identificando tendencias de investigación y temas de controversia. La metodología está basada en el análisis de tres bases de datos especializadas tales como Scopus, Web of Science y Publish or Perish, de las cuales se obtuvieron datos y documentos para evaluar su calidad y contenido. Los resultados determinan el surgimiento de elementos que involucran conceptos, prácticas y críticas asociadas a la co-creación de valor. También emerge una discusión alrededor del término co-tendencias, que conlleva a ampliar la mirada de este proceso del marketing en otras situaciones y actores intervinientes en los sistemas de servicio. Las asociaciones realizadas permiten identificar nuevos enfoques en la aplicación del marketing para una efectiva gestión de las organizaciones. Finalmente, se concluye sobre la necesidad de encontrar nuevos mecanismos para la aplicación y significación de la co-creación de valor teniendo en cuenta la participación de distintos stakeholders.

Palabras clave: co-creación de valor, revisión sistemática de literatura, co-tendencias, prácticas de co-creación, críticas en co-creación de valor.
The purpose of this article is to perform a systematic review of documents to characterize the use of statistical analysis around the value co-creation in order to find common patters and divergent aspects, identifying research trends and controversial issues. The methodology is based on the analysis of three specialized databases such as Scopus, Web of Science and Publish or Perish, from which the data and documents were obtained to evaluate their quality and content. The results determine the emergence of elements that involve concepts, practices and critical issues associated with value co-creation. Evenly, a discussion emerges around the term suggested as co-trend, which entails broadening the view of this marketing process in other situations and actors involved in service systems. The associations made allow to identify new approaches in the applications of marketing for an effective management in organizations. The conclusion focus around the need to find new mechanisms for the application and significance of value co-creation taking into account the participation of the different stakeholders.

Keywords: value co-creation, systematic literature review; cotrends; critical issues on value co-creation. 


\section{INTRODUCCIÓN}

La co-creación de valor surge como respuesta a las dinámicas cambiantes del mercado convirtiéndose en una herramienta flexible de acuerdo al tipo de organización y su contexto, con el fin de generar construcción conjunta de valor, no solamente entre ésta y el cliente, sino también con otros stakeholders como proveedores, distribuidores, competidores y socios, entre otros (Grönroos, 2015, Prahalad \& Ramaswamy, 2000, 2003, 2004a). De esta manera puede convertirse en un proceso complejo de gestión con implicaciones y usos que hacen entender a las organizaciones la forma de gerenciar estratégicamente sus relaciones con el cliente y demás públicos, enriqueciendo tendencias empresariales, denominadas en este trabajo "co-tendencias", como la innovación, el comportamiento del consumidor desde sus niveles de involucramiento y la investigación en sistemas de servicio (Ranjan \& Read, 2014, Edvardsson, Skålén, \& Tronvoll, 2012). No obstante, han surgido quienes han controvertido o entrado en conflicto con las propuestas y alcances hasta ahora dados por la co-creación de valor, pues la perciben como algo metafórico, sin sustancia, encarcelada en su propio concepto, mientras no trascienda en una comprensión más profunda de lo que es la interacción, no únicamente con su target, sino con situaciones y otros actores que hacen parte de los procesos en las organizaciones (Grönroos, 2011; Grönroos \& Gummerus, 2014).

Polese, Mele, \& Gummesson (2017) exhortan hacia el desarrollo de nuevos marcos para analizar la co-creación de valor, como proceso resultante de un cúmulo de relaciones e interacciones entre todos los públicos involucrados, es decir, más que una relación diádica entre organización y cliente, son redes de interacción que necesitan un enfoque holístico e integrador, que requieren ser estudiadas de manera profunda. Adicionalmente, tanto las empresas como los clientes se encuentran en una fase contemporánea en la que hay innovadoras formas para co-crear valor (Saarijärvi, 2012).

Lo anterior cobra mayor sentido cuando Ranjan \& Read, (2014) arguyen que como el trabajo teórico y empírico sobre co-creación de valor ha crecido en distintas direcciones, las raíces teóricas se han vuelto más ambiguas refiriendo incluso que, autores como McColl-Kennedy, Vargo, Dagger, Sweeney, \& van Kasteren (2012) han catalogado hasta 27 definiciones diferentes de este concepto, donde 22 de ellas pertenecen al período posterior del nuevo milenio.

Debido a la situación expuesta, fue que Ranjan \& Read, (2014) emprendieron un proceso de clasificación para determinar las dimensiones teóricas de la co-creación de valor -apoyándose en la literatura existente- llegando a concluir que son: la co-producción y el valor de uso, cada una de estas dimensiones con los siguientes tres elementos subyacentes (categorías): Intercambio de conocimiento, Equidad e Interacción para la primera y, Experiencia, Personalización y Vínculo relacional para la segunda. Investigación que se hizo bajo un paradigma funcionalista y recurriendo al positivismo lógico, ya que pudieron establecer con constructos de medición empíricos, la correlación entre las diferentes variables estudiadas.

Frow, Nenonen, Payne, \& ..., (2015) realizaron aportes teóricos y prácticos a la co-creación de valor a partir de la utilización del método denominado análisis morfológico, el cual les permitió diseñar una versión de diseño marco con seis dimensiones (motivo de co-creación, forma de co-creación, actor comprometido, plataforma de participación, nivel de compromiso, duración del compromiso), a través de la estructuración y análisis de relaciones entre un cúmulo amplio de dimensiones y categorías complejas no cuantificables.

El presente artículo tiene como objetivo presentar, en una primera instancia, las asociaciones conceptuales, prácticas y críticas en torno a la co-creación de valor, a través de una RSL explicada en el ítem 
perteneciente a la metodología, para luego dar paso a la discusión de resultados vistos desde las asociaciones de tendencias, denominadas "co-tendencias". Finalmente, se realizan las respectivas conclusiones y las futuras investigaciones que pueden ser trabajadas en el campo de la co-creación de valor.

\section{METODOLOGÍA}

Para la construcción del presente artículo se utilizó la metodología de revisión sistemática de literatura (RSL) con el propósito de resumir, compilar, criticar y sintetizar la investigación existente sobre el tema a abordar y así analizar todos los datos relevantes, empíricos o teóricos, necesarios para dar respuesta a las preguntas de investigación planteadas (Suter, 2013; Bolderston, 2008). Los objetivos de esta metodología empleada se enfocan en el conocimiento del estado del arte, la identificación de tendencias de investigación y, asimismo, la identificación de puntos de controversia (Suter, 2013), (Jadika, Khoo, \& Na, 2011).

En cuanto a la tipología usada para la RSL, se empleó una preparación de revisión completa, integradora, narrativa y sistemática (Pautasso, 2013; Murphy, 2012), con el fin de encontrar patrones comunes y puntos de divergencia (Pautasso, 2013) para describir cualitativamente las revisiones en combinación con datos estadísticos (Murphy, 2012).

Lo anterior se sistematizó en una plantilla donde se llevó a cabo el planeamiento y ejecución de la RSL, a través de la utilización de tres bases de datos especializadas, Scopus, Web of Science y Publish or Perish (Google Scholar), en las cuales se ingresó la respectiva ecuación de búsqueda con el propósito de identificar documentos similares pertenecientes al campo estudiado, en este caso: co-creación de valor. De dicha búsqueda surgieron los documentos apropiados, de los cuales se llevaron a cabo las respectivas revisiones y se analizaron estadísticamente para determinar frecuencias específicas en aspectos como autores, años, citaciones y fuentes, entre otros (Randolph, 2009).

Finalmente, se efectuó la selección de documentos pertinentes y la evaluación de calidad a través de los diferentes abstracts, verificando además, por medio del indicador de impacto de revistas SCImago Journal \& Country Rank, la validez y confianza de las publicaciones (www.scimagojr.com). Para ello se identificó, para cada fuente, el país al que corresponde, el cuartil en el que se ubica y el índice "h" que posee.

\section{RESULTADOS}

A continuación de describen las fases ejecutadas a lo largo de la investigación, iniciando con las revisiones bibliográficas y referenciales realizadas en las diversas bases de datos objeto de estudio.

\section{DATOS OBTENIDOS EN SCOPUS}

La ecuación de búsqueda específica utilizada en esta base de datos fue:

"value co-creation" OR "co-creation of value" OR "value-in-use" OR "value-in-interchange" OR "service-dominant logic" OR "service dominant logic" OR "customer experience" OR "customer co-creation" OR "firm co-creation" OR "co-creation marketing” OR "marketing co-creation” AND organizations OR organisations

De esta manera se obtuvieron 9270 datos, a los cuales se les aplicaron los filtros relacionados con años de publicación, temáticas e idioma entre otros, arrojando un total de 67 documentos. A continuación, se presentan las estadísticas de estos datos. 


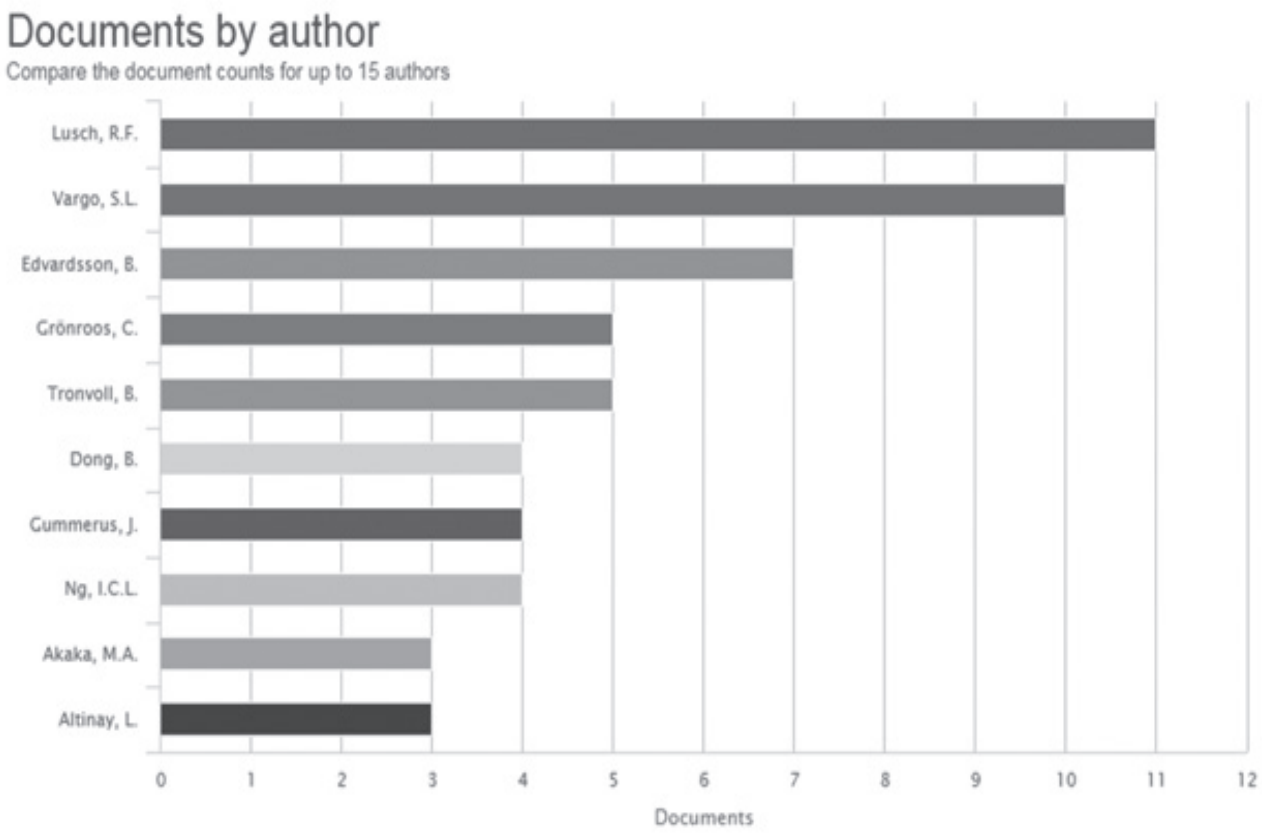

Figura 1 - Documentos por autor

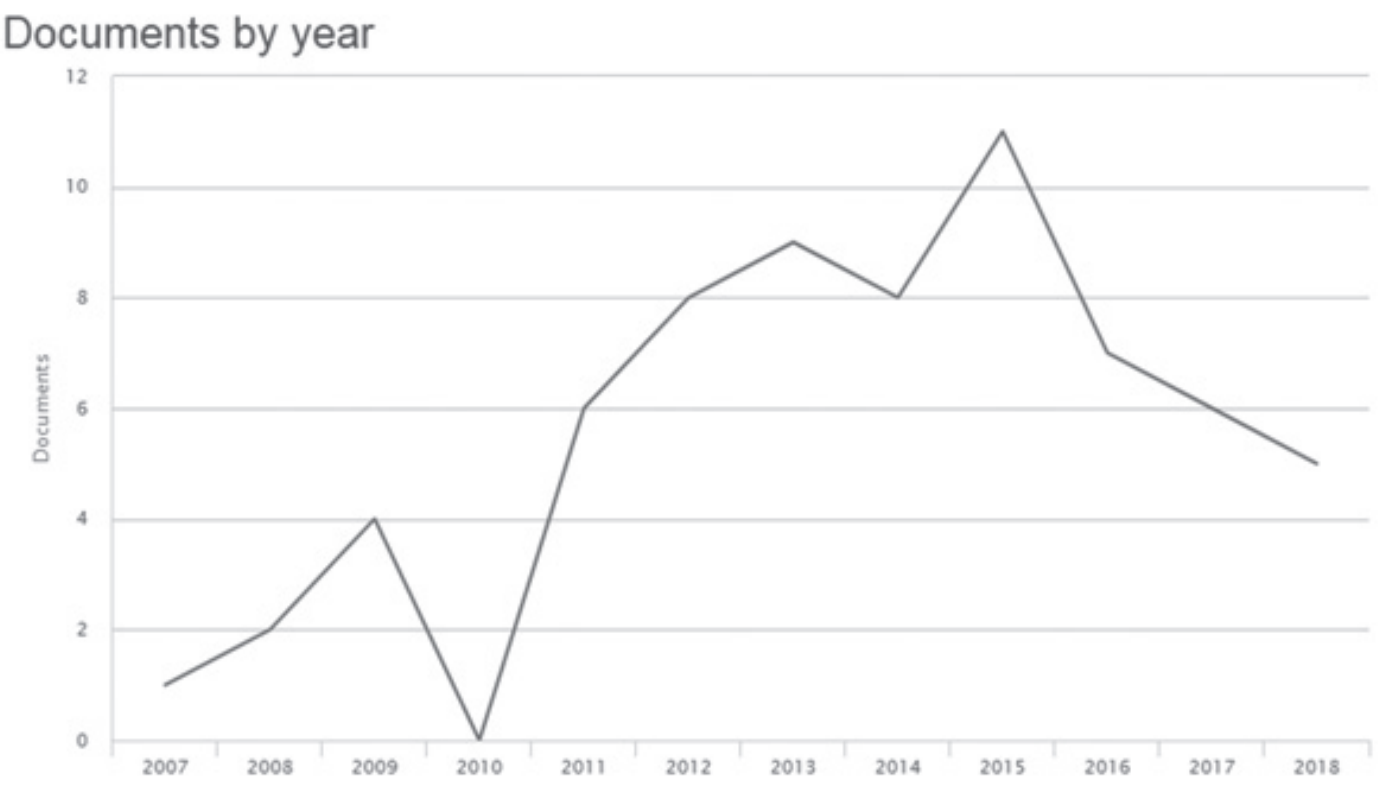

Figura 2 - Documentos por año

La figura 1 permite conocer que entre los autores con mayor número de publicaciones en el tema de co-creación de valor son Stephen $\mathrm{L}$. Vargo, Robert Lusch, Bo Edvardsson y Chris- tian Grönroos. La figura 2 muestra que los años más activos en publicaciones han sido 2015, 2013 y 2014, respectivamente. 


\section{Source documents by year}

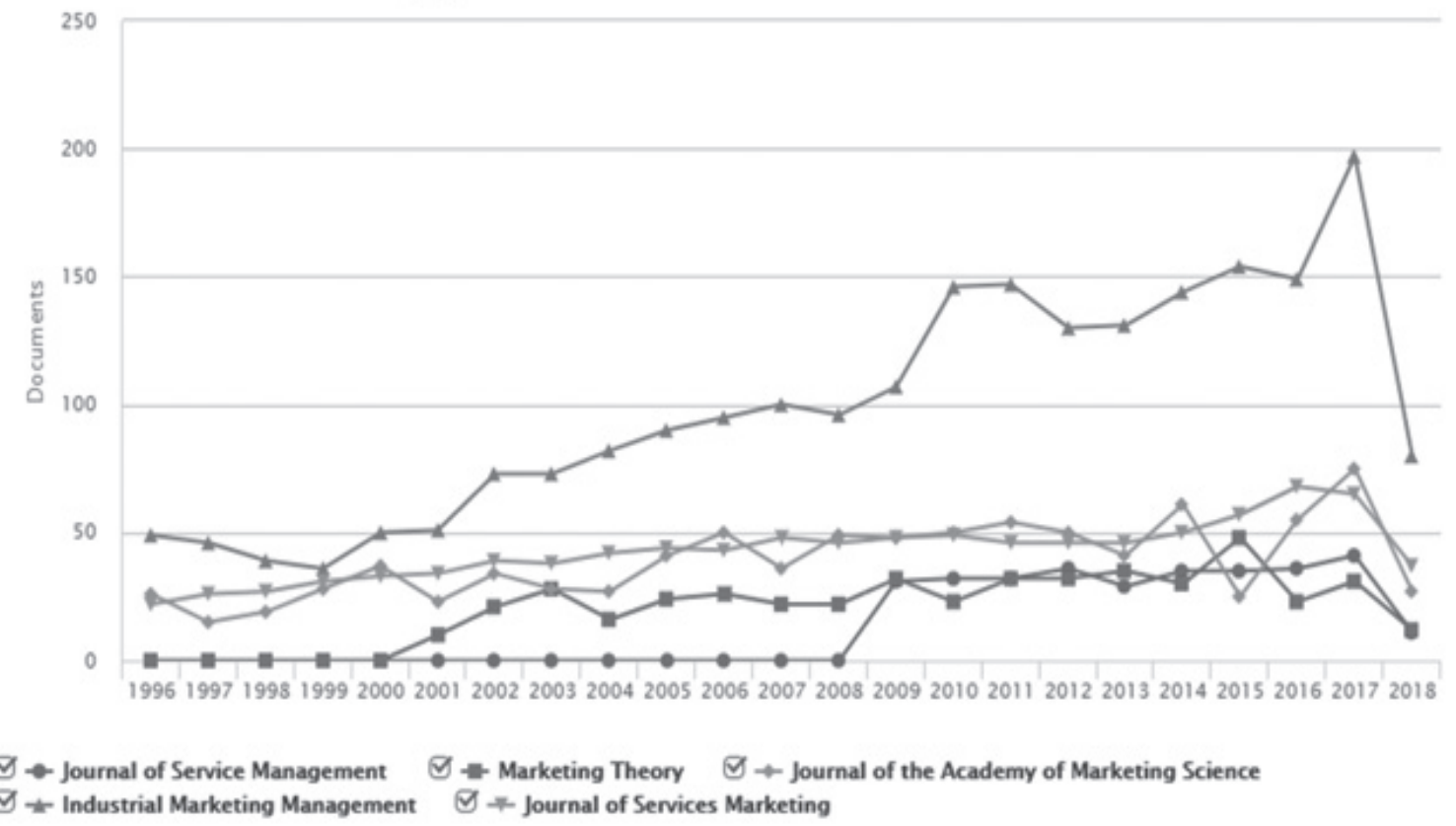

Figura 3 - Fuentes por año

\section{Documents by country/territory}

Compare the document counts for up to 15 countries/territories

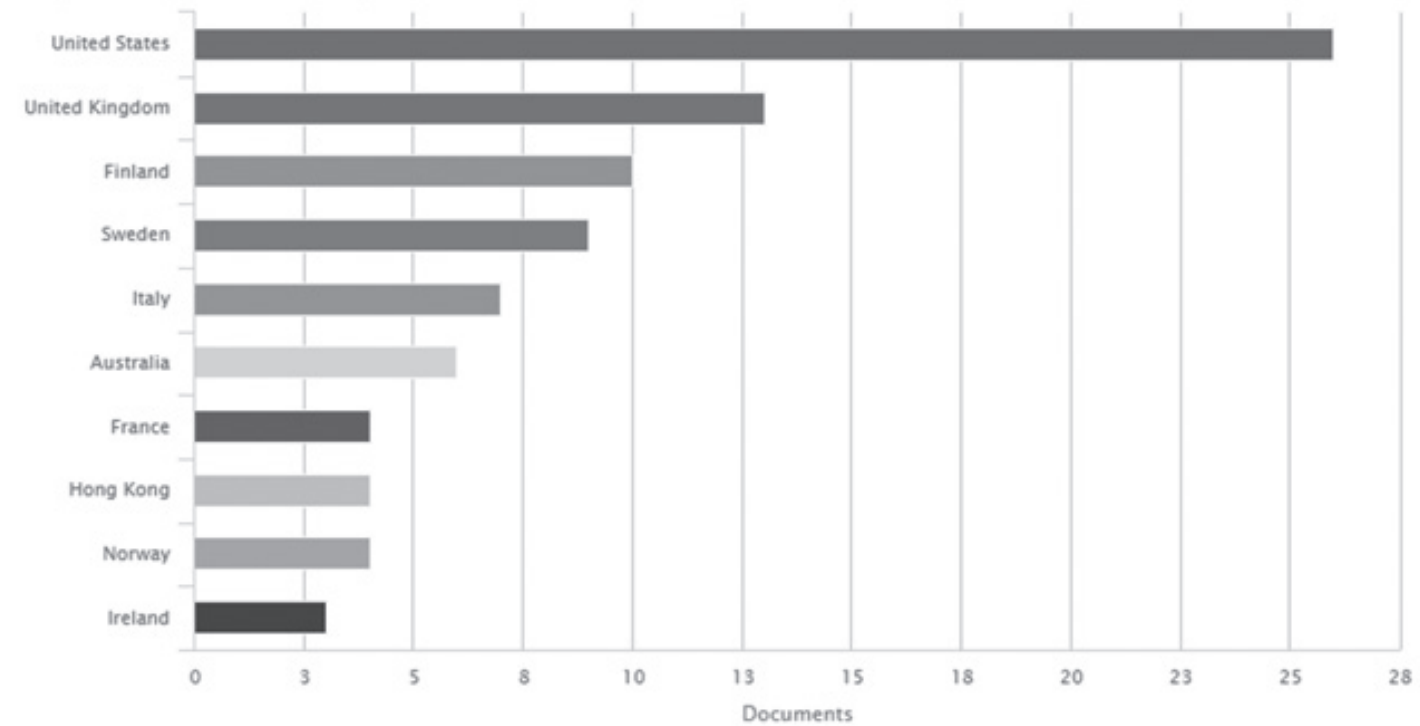

Figura 4 - Documentos por país 
La figura 3 ilustra que dentro de las fuentes más destacadas de publicación de artículos relacionados con el tema de co-creación de valor, se destaca el Journal "Industrial Marketing Management" desde inicios del nuevo mileno. La figura 4 indica que Estados Unidos y Reino Unido son los países de mayor publicación en dicho tema.

\section{Documents by type}

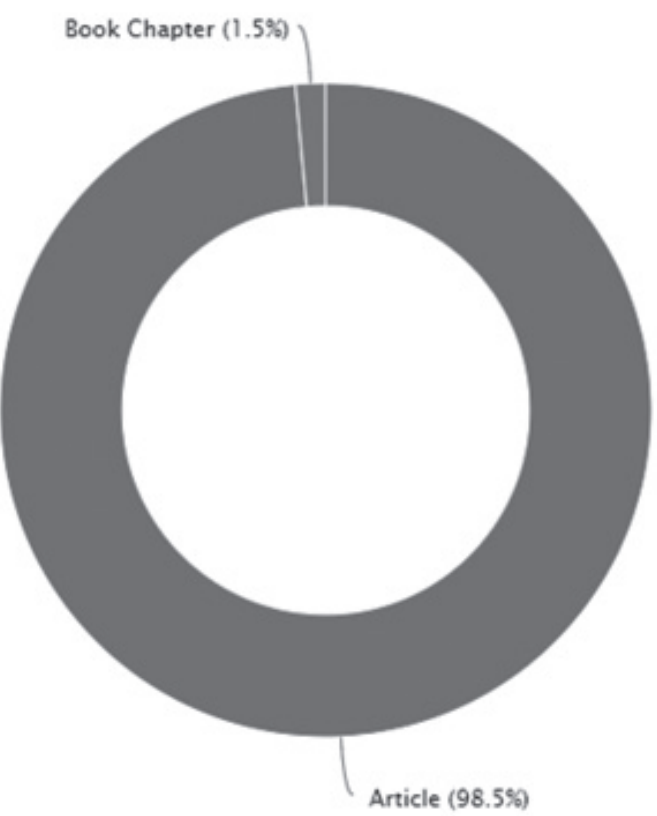

Figura 5 - Tipo de documento

\section{Documents by subject area}

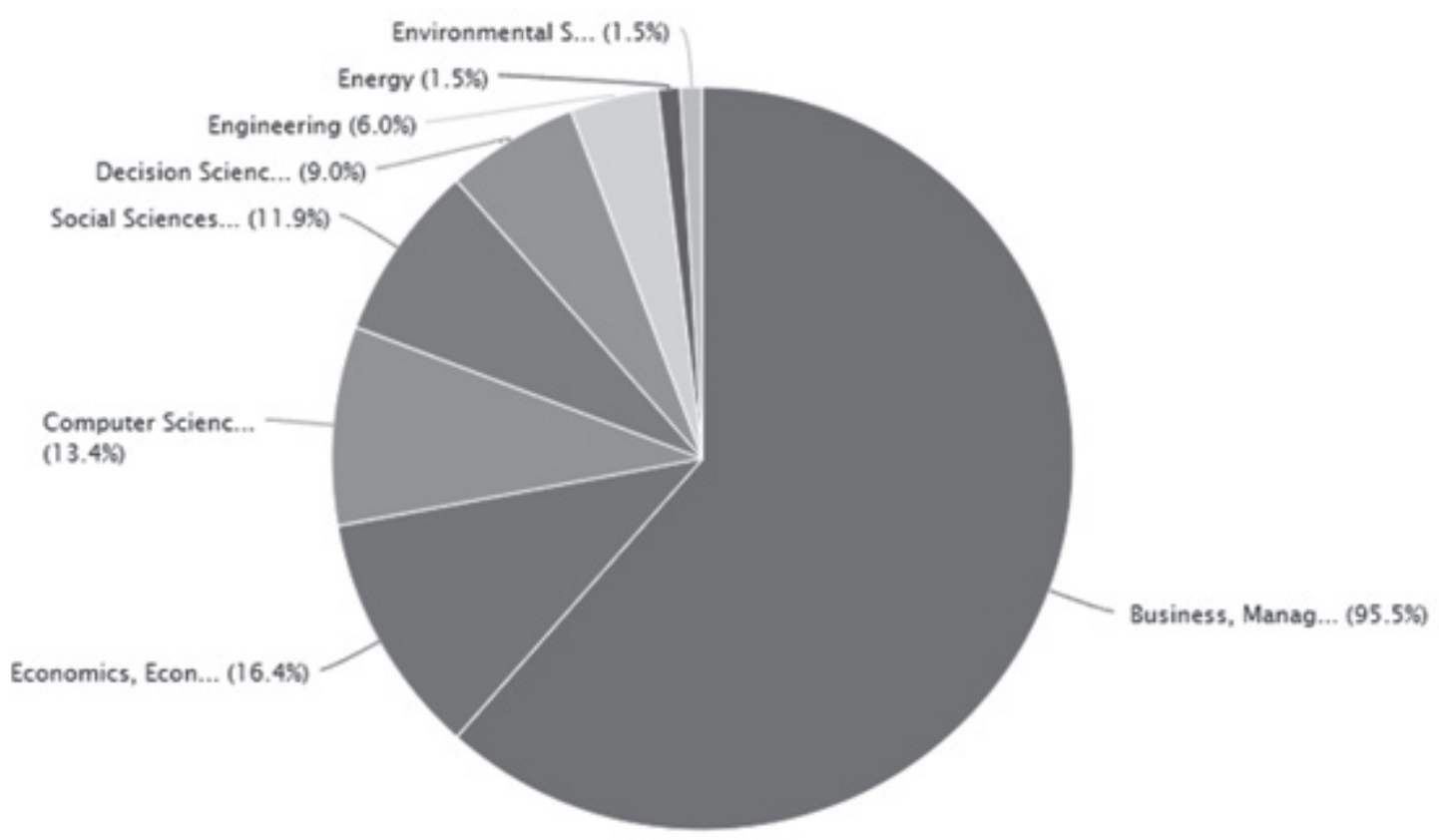

Figura 6 - Áreas temáticas 
La figura 5 expone que el tipo de documento más común es el artículo, mientras que la figura 6 hace ver que el área sobre la que más se escribe en co-creación de valor es Business y Management.

En síntesis, de los 67 documentos se seleccionaron los 36 más pertinentes para el tema.

\section{DATOS OBTENIDOS EN WEB OF SCIENCE}

La ecuación de búsqueda específica utilizada en esta base de datos fue: "value co-creation" OR "co-creation of value" OR "value-in-use" OR "service-dominant logic" OR "service dominant logic" AND organizations

De esta manera se obtuvieron 1016 datos, a los cuales se les aplicaron los Las figuras 7 y 8 señalan el número de elementos publicados por año y las citas cada año, entre los que se destacan 2011 y 2016 con más publicaciones y, 2016 y 2017 con más de 750 citas. filtros relacionados con años de publicación, temáticas e idioma entre otros, arrojando un total de 50 documentos. A continuación, se presentan las estadísticas de estos datos.

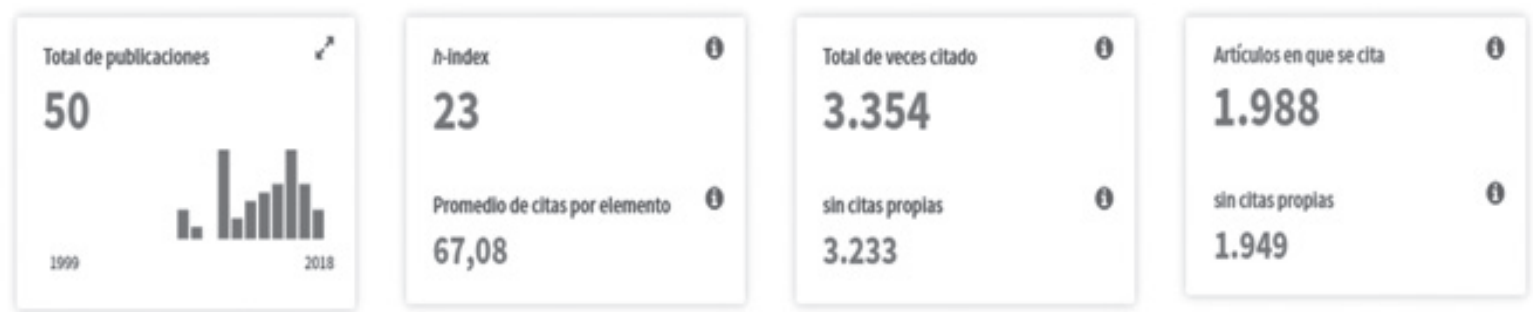

Figura 7 - Estadísticas generales arrojadas por Web of Science de los 50 resultados finales

La figura 7 apunta a que, del total de publicaciones seleccionadas se tiene un índice $h$ equivalente a 23 , con un promedio

Elementos publicados cada año

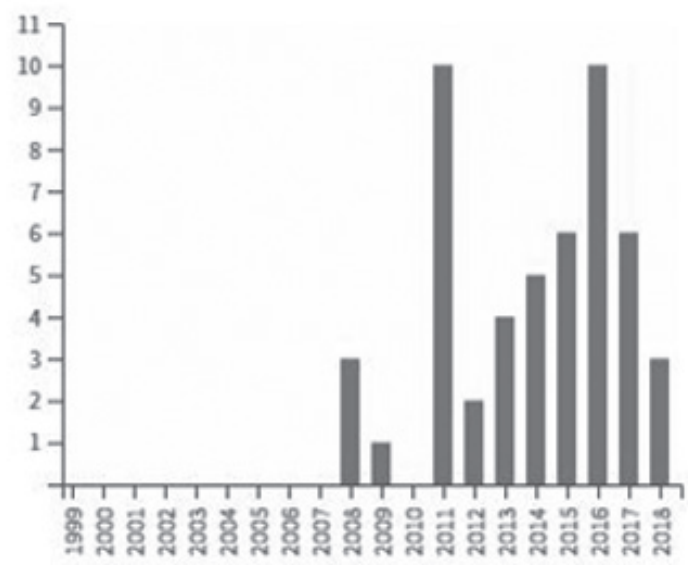

Figura 8 - Elementos publicados por año de citas de 67,08 por elemento, un total de 3.354 veces citado y un total de 1.988 artículos en que se cita.

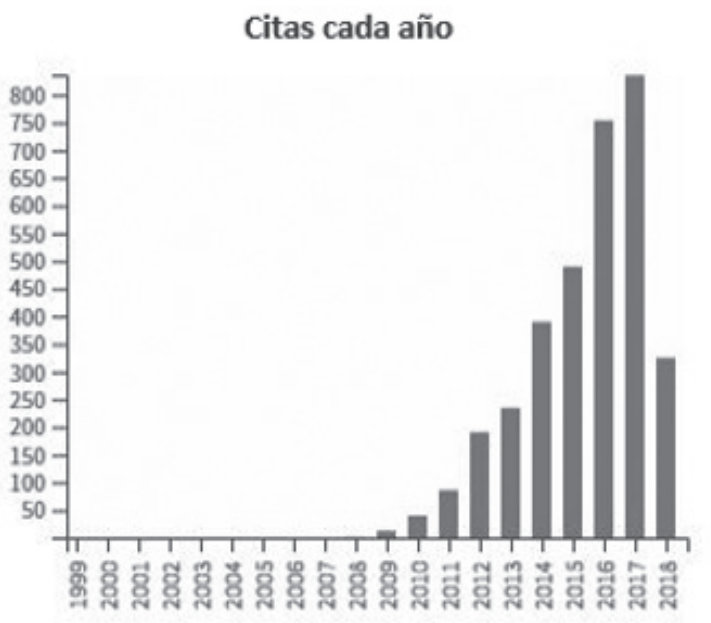

Figura 9 - Número de citas por año 
Las figuras 7 y 8 señalan el número de elementos publicados por año y las citas cada año, entre los que se destacan 2011 y
2016 con más publicaciones y, 2016 y 2017 con más de 750 citas.

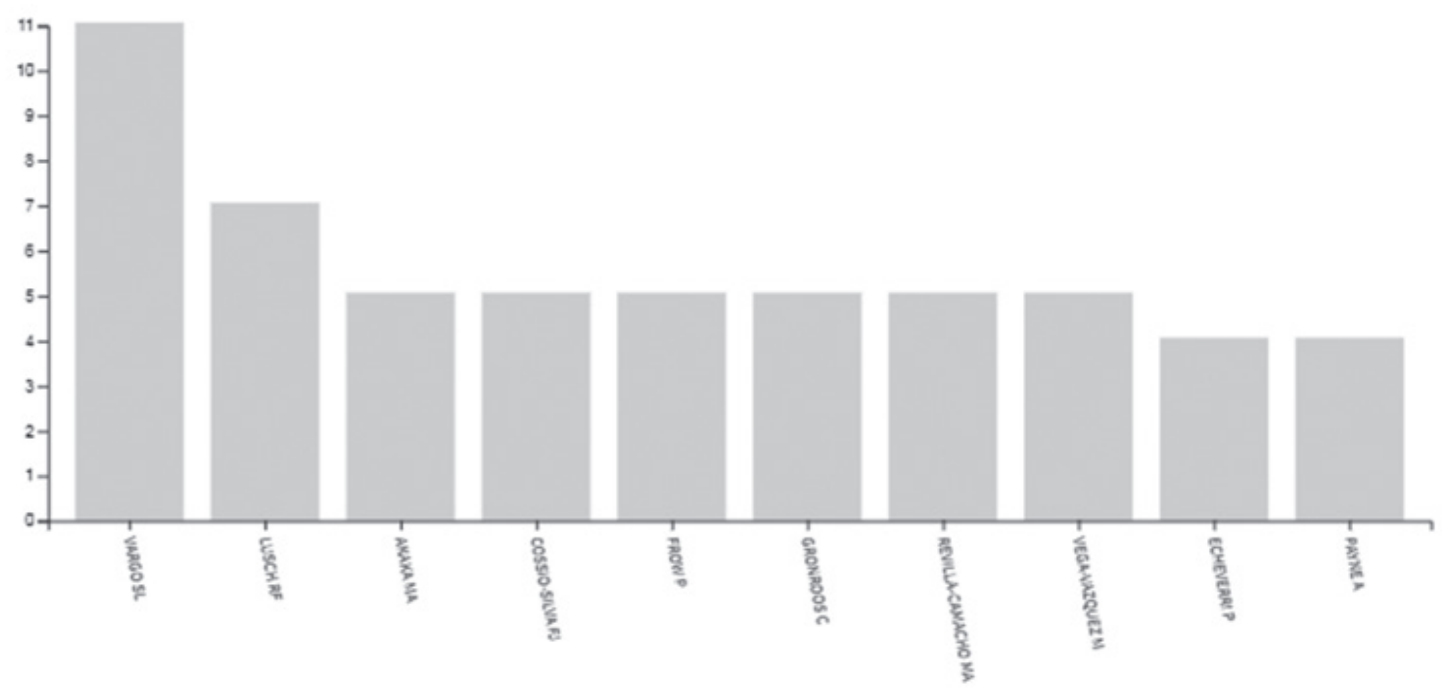

Figura 10 - Autores principales

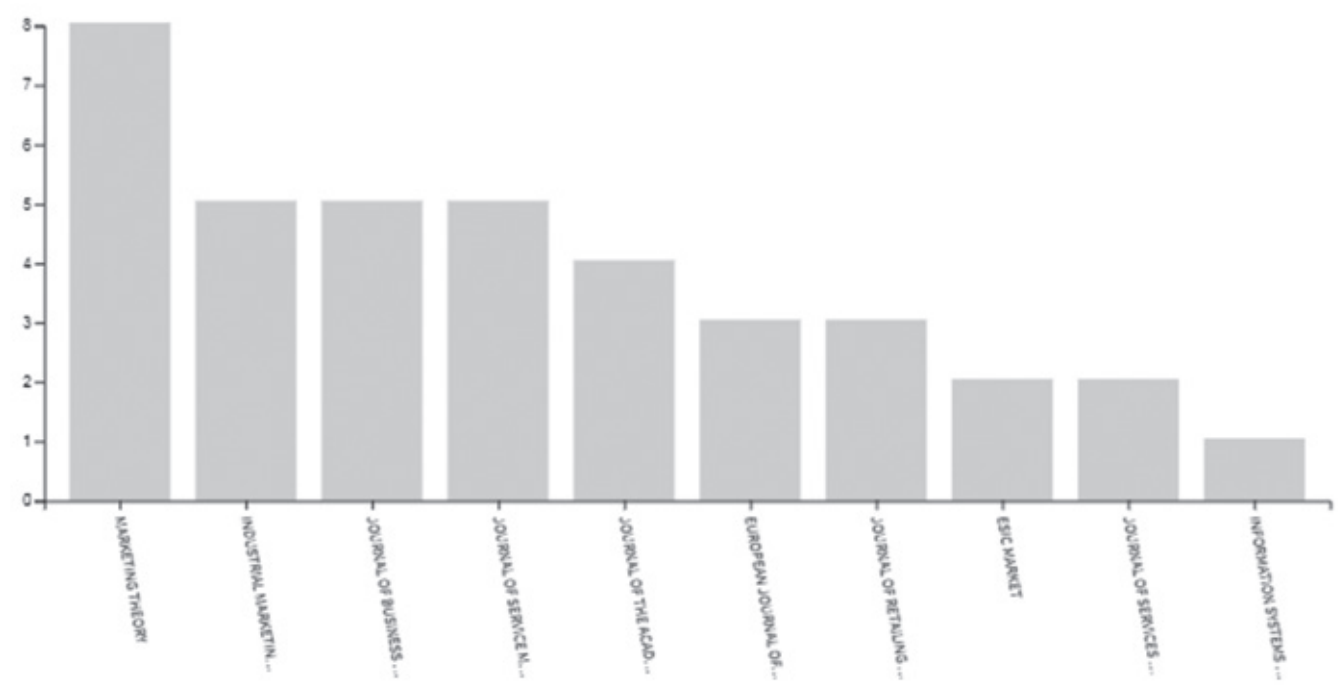

Figura 11 - Fuentes principales

as figuras $10 \mathrm{y} 11$ indican que entre los autores con mayor número de publicaciones en el tema de co-creación de valor están Stephen L. Vargo, Robert Lusch, y Christian Grönroos, mientras que entre las principales fuentes están Marketing
Theory, Industrial Marketing Management y Journal Business Research.

Sintetizando, de los 50 documentos se seleccionaron los 31 más pertinentes para el tema. 


\section{DATOS OBTENIDOS EN PUBLISH OR PERISH (GOOGLE SCHOLAR)}

La ecuación de búsqueda específica utilizada en esta base de datos fue:

"value co-creation" OR "co-creation of value"

De esta manera se obtuvieron 999 datos, a los cuales se eligieron los 15 más citados para efectos de la estadística, la cual se muestra a continuación.

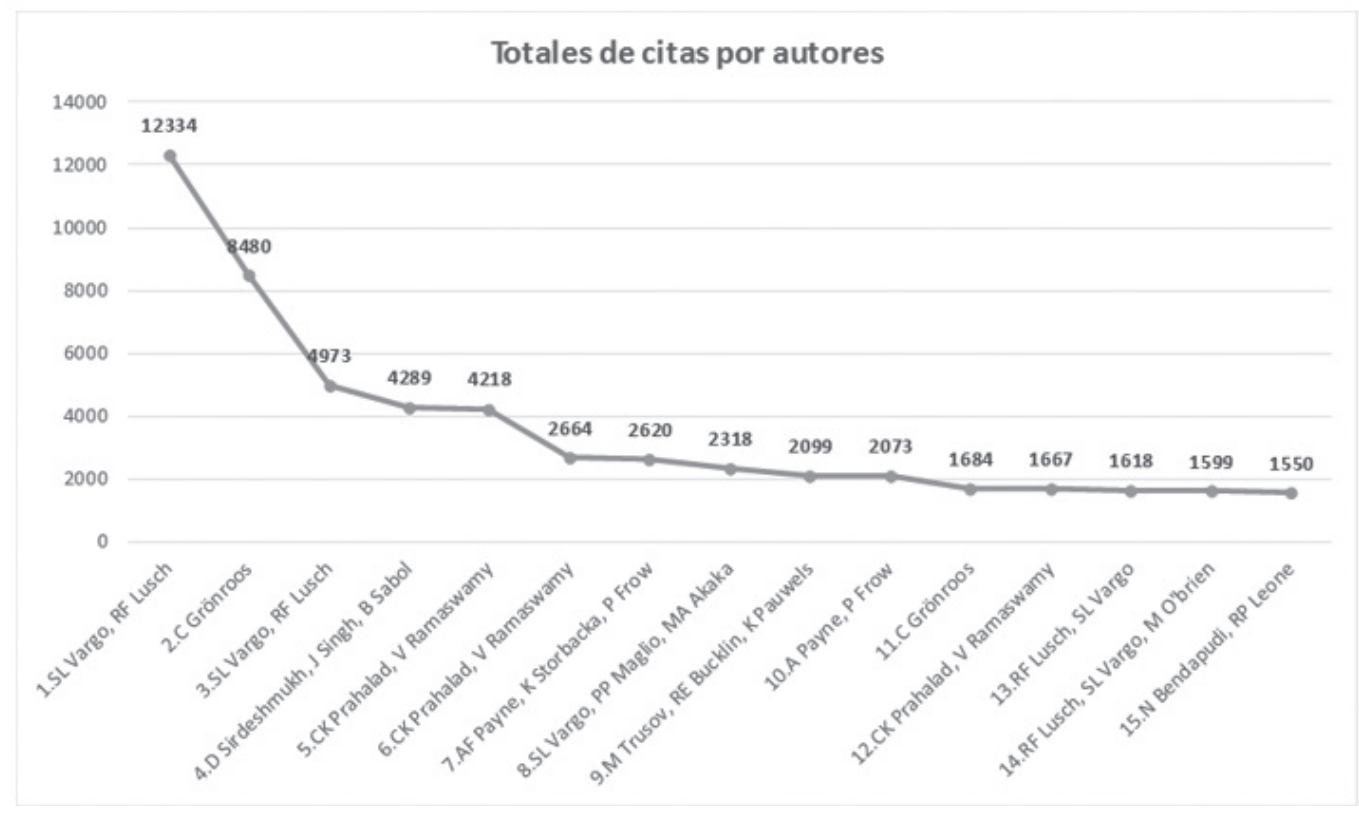

Figura 12 - Citas por autor

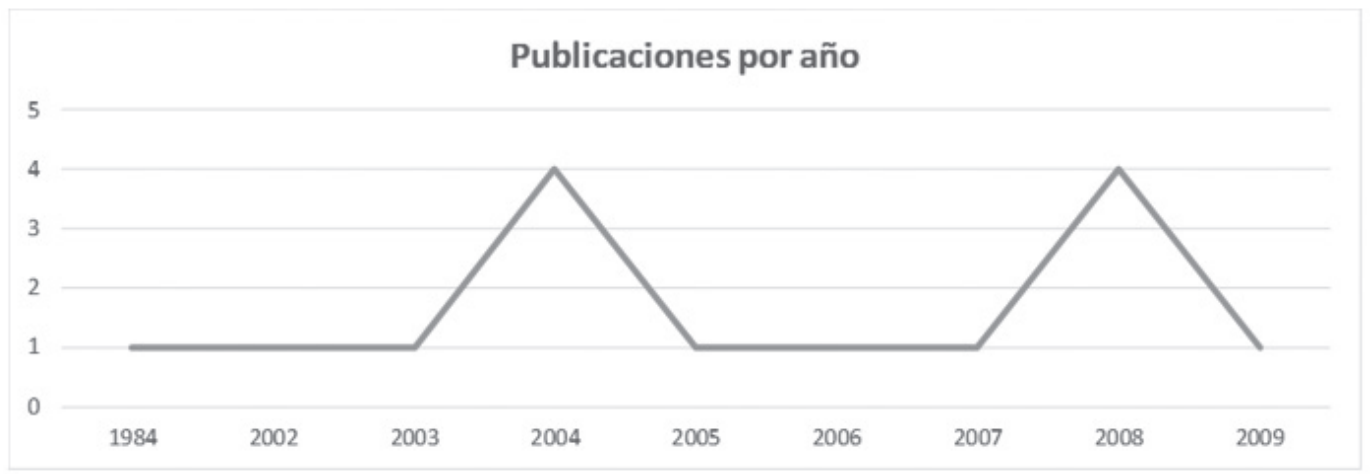

Figura 13 - Publicaciones por año

La figura 12 muestra que entre los autores con mayor número de citaciones están Stephen L. Vargo, Robert Lusch, y Chris- tian Grönroos. Mientras que la figura 13 ilustra que son los años 2004 y 2008 los que mayor número de publicaciones poseen. 


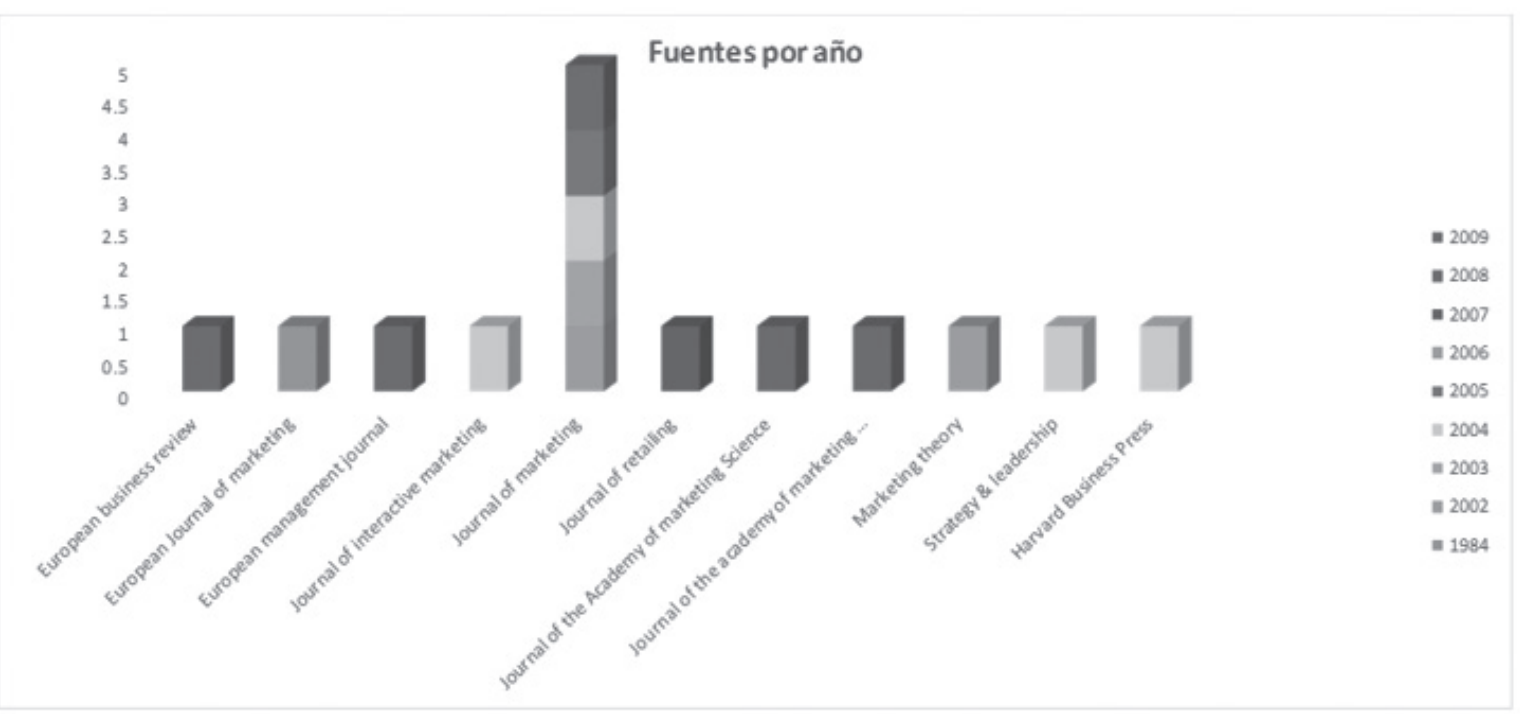

Figura 14 - Publicación por año/fuente

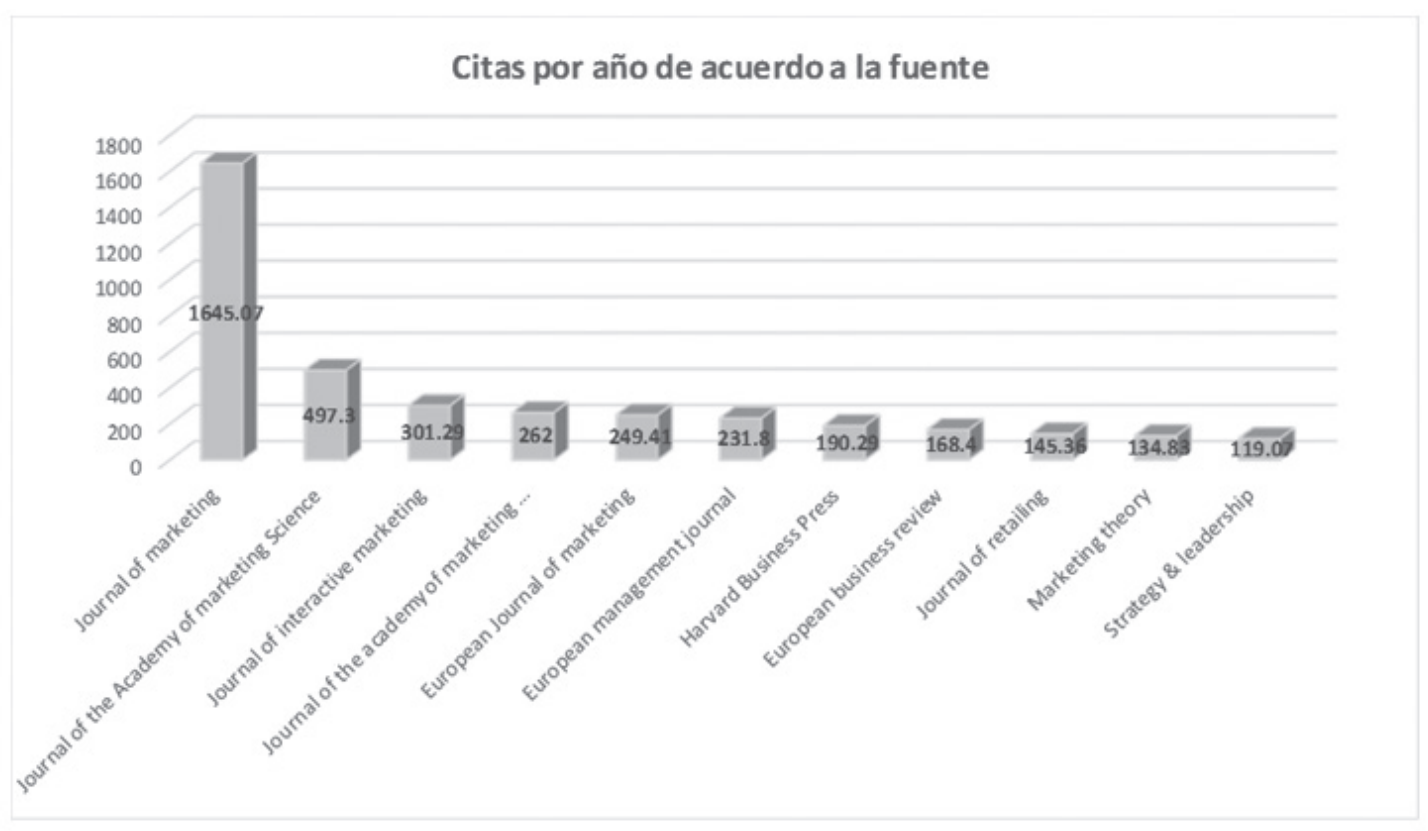

Figura 15 - Cita por año / fuente

La figura 14 señala que Marketing Theory es la fuente más destacada en temas de co-creación de valor en los años 2002 a 2009. Y la figura 15 hace ver que el Jornal of Marketing es la fuente con mayor número de citaciones por año, seguida por el Journal of the Academy of Marketing Science.

Por último, de los 999 documentos, se eligieron los 40 más pertinentes para la selección y evaluación de calidad.

Es importante aclarar que, para efectos de obtención de mejores resultados, la ecua- ción de búsqueda se fue adaptando por la mejor opción, de acuerdo a la base de datos utilizada, motivo por el cual el número de conceptos utilizados en Scopus es mayor a los usados en Web of Science y en Publish or Perish; no obstante, así se pudo manejar un menor número; además, no hubo cambios o ajustes de palabras, con el fin de mantener homogeneidad en la información de entrada.

Se muestra seguidamente en la tabla 1 la construcción de la estadística obtenida a través de SCImago Journal \& Country Rank, ya que las fuentes que predomina- 
ron en las tres bases de datos utilizadas para la RSL, fueron sometidas a una búsqueda de información con el fin de deter- minar la calidad de las publicaciones seleccionadas.

Tabla 1: Ranking de las publicaciones seleccionadas en la RSL

\begin{tabular}{|ccccc|}
\hline No. & Fuente & País & Cuartil & Índice $\mathrm{h}$ \\
\hline 1 & Journal of Marketing & Estados Unidos & Q1 & 208 \\
\hline 2 & Journal of Business Research & Países Bajos & Q1 & 144 \\
\hline 3 & $\begin{array}{c}\text { Journal of the Academy of } \\
\text { Marketing Science }\end{array}$ & Estados Unidos & Q1 & 139 \\
\hline 4 & Journal of Retailing & Reino Unido & Q1 & 111 \\
\hline 5 & Industrial Marketing Management & Países Bajos & Q1 & 106 \\
\hline 6 & European Management Journal & Reino Unido & Q1 & 84 \\
\hline 7 & Journal of Services Marketing & Reino Unido & Q1 & 71 \\
\hline 8 & European Journal of Marketing & Reino Unido & Q1 & 44 \\
\hline 9 & Marketing Theory & Reino Unido & Q1 & 40 \\
\hline 10 & Journal of Service Management & Reino Unido & Q1 & 74 \\
\hline
\end{tabular}

Fuente: Elaboración propia con base en SJR (https://www.scimagojr.com/

ASOCIACIONES CONCEPTUALES

En la revisión de literatura sobre la co-creación de valor se encuentran varias precisiones realizadas por autores relevantes en el campo del marketing, las cuales comenzaron a surgir en la primera década del nuevo milenio; no obstante, es de resaltar que desde el S. XX en los años go ya Crönroos (2015) interesado por el papel incipiente del marketing -caracterizado hasta ese momento por intercambios con transacciones aisladas sin tener en cuenta la construcción de relaciones a largo plazo con los clientes-, resaltó sus bondades y alcances haciendo reflexiones en cuanto a que debía considerarse como un aspecto integral de la estrategia en una organización, donde era fundamental incluir varios actores cuyos objetivos debían ser cumplidos a través de una interacción constante con sus stakeholders, quienes han pasado de un rol pasivo a uno activo, ganando una mayor relevancia (Prahalad \& Ramaswamy, 2000).

Dicha relevancia se hizo evidente a través del servicio, por los procesos de intercambio generados entre compradores y vendedores, dando lugar a una transformación desde lo transaccional hasta lo relacional. Fue Gummesson (2012), quien sugirió tres paradigmas acerca de la evolución que ha tenido el marketing en cuanto al servicio: el pri- mero, anterior a la década de los 70, donde el servicio aún no era reconocido como una economía productiva porque prevalecían los bienes y la manufactura, es decir la tangibilidad. El segundo, dado entre la década de los 70 hasta el año 2000, identificado por el establecimiento de las diferencias entre bienes y servicios, pues ya se reconocía a estos últimos como una actividad económica fundamental, incluso como complemento para la comercialización de productos tangibles. Estos dos paradigmas conllevan por tanto a orientaciones hacia el producto y hacia el cliente, es decir, un enfoque más desde la empresa que desde el target mismo. Y el tercero, desde el inicio del nuevo milenio en adelante, el cual -a diferencia del segundo paradigma- da entrada a una era encauzada a las similitudes e interdependencias entre los bienes y los servicios, generando con ello un cambio de perspectiva desde la lógica dominante del marketing, es decir que, el enfoque desde el intercambio pasa a ser de valor tangible a intangible.

Con base en esto, fueron Vargo \& Lusch $(2004,2008)$ quienes argumentaron que en las últimas décadas esa nueva lógica dominante para el marketing, denominada lógica dominante del servicio -DS-, es la que supera las limitaciones de la dinámica tradicional de los bienes al centrarse en los recursos intangibles, en las relaciones y en la crea- 
ción conjunta de valor, razones substanciales para el intercambio económico. De esta manera, la interacción entre la empresa y el cliente se convierte en el lugar de creación de valor (Prahalad \& Ramaswamy, 2004b). De hecho, Vargo \& Akaka (2009) argumentan que no hay "servicios", sino "servicio" como el acto de hacer algo por la contraparte y como la base del intercambio mismo, por ende, sostienen que el valor siempre será co-creado. Esto último es crucial como punto central de esta lógica dominante desde el servicio, pues en ella se aduce que el valor se co-crea, es decir se crea en conjunto, en colaboración, convirtiéndose para el cliente en una experiencia vívida y única. Es por lo anterior que, Vargo \& Lusch (2008) afirman que la empresa es incapaz de crear valor de manera unilateral, pero si puede orientar un proceso que requiere la participación del beneficiario en la co-creación de valor, lo cual es posible gracias al diálogo y a la interacción permanente.

De otro lado, adherido a la co-creación, emerge la importancia de la experiencia del cliente, donde Prahalad \& Ramaswamy, (2004a) exponen que ésta es la base del valor. Para ello, establecen un paralelo entre un mercado como target principal y un mercado orientado a la co-creación de experiencias. Entre ambos la principal diferencia es que el primero se caracteriza por una separación evidente entre el consumidor y la empresa, haciendo que ésta quiera ser la dueña de la relación convirtiendo a su grupo objetivo en una especie de "presa"; mientras que en el segundo existe una convergencia de los roles y, por tanto, el valor es co-creado en los múltiples puntos de contacto e interacción. Ahora, dependiendo de la calidad que estos puntos tengan, será decisivo para generar, incluso, nuevas fuentes de ventaja competitiva, pero teniendo en cuenta el papel activo que juega el cliente, pues de lo contrario existirá un desequilibrio en el tipo de experiencia que se desea construir.

Para Meyer \& Schwager (2007) la experiencia del cliente es la respuesta interna y subjetiva que éste tiene ante cualquier contacto directo o indirecto con una empresa. Explican que contacto directo es el que tiene que ver con el transcurso de la compra, la utilización y el servicio, procedido generalmente por el cliente; mientras que el indirecto se refiere a contactos no planificados, pero que a menudo se dan a través del "voz a voz" y la publicidad, por mencionar algunos. De esto se interpreta que el valor está presente en las experiencias que viven los clientes, más no en el consumo de los productos (Prahalad \& Ramaswamy, 2004b), y es por ello que, las organizaciones deben propender por la existencia de múltiples y diversas interacciones que desemboquen en una co-creación de valor, donde la experiencia cobra un papel relevante al estimular el rol activo que tiene el consumidor, dando como resultado una negociación en la que el escenario de compra-venta se asumirá de manera proporcionada para los actores intervinientes (cliente-empresa).

Como respaldo a lo expuesto, Andajani, (2015) define la experiencia como el conjunto de los eventos experimentados por los clientes en el proceso antes y después de la compra, siendo esto personal y único, además que estimula los sentidos y los aspectos emocionales, racionales y físicos a fin de crear una experiencia inolvidable; por ende, el consumidor se involucra afectivamente en el proceso de compra. En cuanto a la empresa, es ella quien posee la capacidad para controlar la reacción de los clientes y orientar la obtención de la experiencia tal y como lo espera, pues es a través de la estimulación que ésta ha planeado cómo logra enfocarse en los sentidos de sus consumidores durante todos los momentos de contacto.

Sin embargo, Verhoef et al., (2009) sostienen que a pesar de ser la experiencia una generadora de co-creación de valor, se adolece de teorías subyacentes en cuanto a antecedentes y consecuencias de esa experiencia del cliente donde haya implicaciones a nivel gerencial, e incluso destacan el trabajo de Pine II \& Gilmore, (1998) en cuanto a que la creación de una experiencia de cliente que sea distintiva, puede proveer un efectivo valor económico para las empresas. Por tanto, Verhoef et al., (2009) construyeron un modelo conceptual de antecedentes y asesores de la experiencia del cliente, evaluando la necesidad de estrategias de gestión en este tema, con el propósito se ser tomados en cuenta por las organizaciones, que les interese más que el resultado y las acciones administrativas, diseñar y aplicar estrategias gerenciales para la experiencia del cliente. 
Iniciando la segunda década del S. XXI fueron Grönroos \& Ravald, (2011) quienes concluyeron que la creación de valor para el cliente consta de dos subprocesos: el del proveedor para proporcionar recursos a sus consumidores, y el del cliente para convertir el servicio en valor. Como resultado, formularon cinco tesis que ayudan a una mayor comprensión de dichos subprocesos, las cuales se enfocan en el marketing como garante de la creación de valor para los clientes, la reciprocidad de la creación de valor entre empresa y target, el cliente como co-creador de valor, el proveedor como facilitador de valor pudiendo convertirse también en co-creador del mismo y, el proveedor de servicios como soporte del cumplimiento de valor a través de las interacciones con sus clientes.

Complementando el aporte de Grönroos \& Ravald, (2011), Edvardsson, Skålén, \& Tronvoll, (2012) van más allá de la dinámica de los subprocesos proveedor y cliente, al introducir una perspectiva sociológica sobre la integración de recursos, es decir que, prestan más atención a las fuerzas sociales y no solamente a los recursos como tales, para que las organizaciones diseñen sistemas de servicio que brinden co-creación de valor a sus stakeholders. Siendo así, se comienza a escalar en las dinámicas de la co-creación de valor, pues ya no es algo que se encuentra inmerso en las esferas propias de las organizaciones, sino que sale de ellas, para imbuirse en estructuras sociales que contextualizan las lógicas emergentes y subyacentes de la relación cliente-empresa. Sin embargo, en el trabajo desarrollado por Hilton, Hughes, \& Chalcraft, (2012) se define el valor como un juicio evaluativo que es netamente personal y que, por tanto, no puede ser co-creado; a este respecto, los autores mencionan que los actores intervinientes ejecutan el valor pero, como resultado de la co-creación de servicio.

Meynhardt, Chandler, \& Strathoff, (2016) plantean nueve principios sistémicos de co-creación de valor, al sostener que el valor es un parámetro de orden surgido en los ecosistemas de servicio. Critican la visión micro-personal o macro-colectiva en la que se han enfocado la mayoría de las investigaciones sobre co-creación de valor, permitiendo que se aíslen elementos conexos, lo cual no pasaría si se desarrollaran desde una perspectiva sistémica o de interrelación. Di- chos principios en una lógica sistémica son: (i) distancia crítica, (ii) estabilidad, (iii) amplificación, (iv) determinación interna), (v) no linealidad y retroalimentación, (vi) transiciones de etapa, (vii) ruptura de simetría, (viii) previsibilidad limitada y, (ix) dependencia histórica. Se identifica también en estos autores una mayor complejidad asociada a la co-creación de valor, la cual sigue incrementando su alcance y elevando su importancia para la gestión de las organizaciones.

Como respuesta a los aportes efectuados por los diferentes autores, sobre todo después de culminada la primera década del nuevo milenio, han sido Vargo \& Lusch, (2017) quienes han visualizado la lógica DS hacia el año 2025, reconociendo las limitaciones que esta teoría ha tenido con respecto a la co-creación de valor, y por tanto, afirman que ha tomado una serie de giros teóricos significativos y ha añadido/ajustado premisas fundamentales. En este sentido, dentro de los resultados de sus procesos investigativos invitan a la comunidad científica a utilizar dicha teoría como un marco más amplio para el estudio del macro-marketing, incluyendo la ética, la sostenibilidad económica-ambiental-social, y las políticas públicas. Por ello, sostienen que la lógica DS continúa su evolución para lograr avanzar hacia un mayor desarrollo de una teoría general de mercado y de co-creación de valor.

\section{ASOCIACIONES PRÁCTICAS}

Debido al alto nivel de praxis que conlleva la co-creación de valor, Vega-Vazquez, Revilla-Camacho, \& Cossío-Silva, (2013) argumentan que la literatura académica, en su mayor parte, destaca la influencia positiva que se genera en la participación del consumidor en este sentido; sin embargo, aducen que son incipientes los estudios donde se analizan las consecuencias del comportamiento desde la perspectiva del cliente cuando se llevan a cabo procesos de co-creación. En la tabla 2, se muestra una selección de prácticas que han efectuado algunos investigadores en los últimos años, cuyo propósito común fue analizar y comprender los resultados arrojados por los distintos estudios realizados en los actores intervinientes, además de proponer acciones futuras de investigación. 
Tabla 2 - Algunas prácticas de co-creación de valor

\begin{tabular}{|c|c|c|c|}
\hline Sector y Año & Autores & Metodología & Resultados/Conclusiones \\
\hline $\begin{array}{l}\text { Industrial } \\
2008\end{array}$ & $\begin{array}{l}\text { (Cova \& } \\
\text { Salle, } \\
2008 \text { ) }\end{array}$ & $\begin{array}{l}\text { Con base en } 2 \text { estudios de caso } \\
\text { rcsaltaron los límitcs dc las } \\
\text { estrategias de oferta actuales en } \\
\text { términos de co-creación e } \\
\text { involucrando la red de clientes. }\end{array}$ & $\begin{array}{l}\text { Sugirieron un enfoque paso a paso para co-crear } \\
\text { valor en la red de clientes, basándose en un cambio } \\
\text { de la propuesta de valor del cliente a la propuesta de } \\
\text { valor de la red de clientes. }\end{array}$ \\
\hline $\begin{array}{l}\text { Servicios } \\
\text {-Alquiler de } \\
\text { autos } \\
2009\end{array}$ & $\begin{array}{l}\text { (Payne, } \\
\text { Storbacka, } \\
\text { Frow, \& } \\
\text { Knox, } \\
2009 \text { ) }\end{array}$ & $\begin{array}{l}\text { Estudio de caso donde ilustran cómo } \\
\text { la co-creación de valor ayuda en el } \\
\text { diseño y la gestión de la experiencia } \\
\text { con la marca para un producto } \\
\text { innovador. }\end{array}$ & $\begin{array}{l}\text { Invitan a seguir investigando las experiencias de los } \\
\text { clientes para saber cómo puede esto contribuir a la } \\
\text { gestión de la marca en un entorno de SD; aunque } \\
\text { esta lógica esté en una etapa temprana de } \\
\text { desarrollo en la co-creación de valor de marcas. }\end{array}$ \\
\hline $\begin{array}{l}\text { Servicios } \\
\text {-Belleza y } \\
\text { cuidado } \\
\text { personal } \\
2013\end{array}$ & $\begin{array}{l}\text { (Vega- } \\
\text { Vazquez } \\
\text { et al., } \\
2013 \text { ) }\end{array}$ & $\begin{array}{l}\text { Partieron de la medición de co- } \\
\text { creación deede la perepectiva de los } \\
\text { propios clientes para relacionarlo con } \\
\text { su satisfacción. Entrevistaron } 547 \\
\text { usuarios mayores de } 18 \text { años y } \\
\text { analizaron los datos con SPSS y } \\
\text { AMOS. }\end{array}$ & $\begin{array}{l}\text { Con base en el modelo causal empleado, se } \\
\text { confirmó la relación entre el comportamiento de co- } \\
\text { creación de valor y la satisfacción del cliente con la } \\
\text { experiencia del servicio. Sugieren que las empresas } \\
\text { de belleza y cuidado personal fomenten la co- } \\
\text { creación de valor con sus clientes. }\end{array}$ \\
\hline $\begin{array}{l}\text { Servicios } \\
\text { empresariales } \\
2012\end{array}$ & $\begin{array}{l}\text { (Aarikka- } \\
\text { Stenroos } \\
\& \\
\text { Jaakkola, } \\
\text { 2012) }\end{array}$ & $\begin{array}{l}\text { Realizaron } 120 \text { entrevistas } \\
\text { cualitativas con proveedores y } \\
\text { compradores de de servicios } \\
\text { empresariales de conocimiento. El } \\
\text { estudio exploratorio analizó las } \\
\text { actividades, roles y recursos de } \\
\text { ambos actores en el proceso de co- } \\
\text { creación de valor recíproco y sus } \\
\text { implicaciones. }\end{array}$ & $\begin{array}{l}\text { Sugieren que esta experiencia investigativa sirve } \\
\text { como una herramienta de gestión para determinar } \\
\text { los recursos y funciones fundamentales para los } \\
\text { proveedores y clientes, facilitando las actividades } \\
\text { conjuntas y la optimización en la utilización de los } \\
\text { recursos en los servicios empresariales. }\end{array}$ \\
\hline $\begin{array}{l}\text { Entretenimiento } \\
\text {-Juguetes } \\
\text { infantiles LEGO } \\
2014\end{array}$ & $\begin{array}{l}\text { (Akaka et } \\
\text { al., 2014) }\end{array}$ & $\begin{array}{l}\text { Llevaron a cabo } 25 \text { entrevistas en } \\
\text { profundidad a diferentes actores de } \\
\text { la firma, internos y externos, para } \\
\text { evidenciar el papel de los símbolos } \\
\text { en la co-creación de valor en un } \\
\text { ecosistema de servicio. }\end{array}$ & $\begin{array}{l}\text { Argumentan que los símbolos guían a los actores - } \\
\text { internos y externos- en la difusión de prácticas de } \\
\text { co-creación de significados compartidos, ayudando } \\
\text { a determinar el valor de las interacciones presentes } \\
\text { y futuras. }\end{array}$ \\
\hline
\end{tabular}

$\begin{array}{ll}\text { Servicios } & \\ \text {-Tecnologiay } & \text { (Akesson } \\ \text { autoservicio } & \text { et al., } \\ 2014 & 2014 \text { ) }\end{array}$

Hicieron un estudio exploratorio inductivo para examinar en los clientes sus experiencias, al usar un autoservicio con tecnología. Se analizaron 60 entrevistas a clientes categorizando sus respuestas.
Los autores clasificaron en 4 categorías principales, los conductores de la experiencia: informativo, relacional, organizacional y tecnológico. Todos ellos en 3 momentos: antes, durante y después de la visita al autoservicio. También identificaron 13 factores favorables y desfavorables que guían la cocreación de valor para la experiencia del cliente, lo cual puede ser usado por los gerentes de este tipo de tiendas.

La integración de los empleados respaldó 9 propuestas clave, abriendo esto al desarrollo de aportes teóricos, implicaciones gerenciales y nuevos caminos investigativos. Se determinó que los clientes pueden usar 12 tipos de recursos de cocreación de valor; sin embargo, si los empleados no se integran correctamente se da paso a la codestrucción.

$\begin{array}{ll}\begin{array}{l}\text { Servicios } \\ \text { interactivos }\end{array} & \text { (L Plé, } \\ 2016 & \text { 2016) }\end{array}$

El autor centró su estudio en el concepto de participación del cliente para identificar la naturaleza de sus recursos y su integración por parte de los empleados del servicio Llevaron a cabo un ejercicio Los resultados permitieron conocer que en las 5 investigativo exploratorio tomando prácticas de co-creación de valor, basadas en la como caso único la empresa sueca interacción (informar, saludar, cobrar, entregar y Servicios (Echeverri, como caso únicola empresa sueca $\begin{array}{llll}\text {-Transporte } & \text { Skălén, \& } & \text { de transporte público "Gotemburgo } \\ \text { Skálen, } & \text { Tramways" (GT), con el fin de }\end{array}$ público 2011) centrarse en las interacciones entre los conductores y viajeros del sistema. ayudar), se presentaba dicha co-creación cuando los elementos eran congruentes para ambos actores (conductores y viajeros), pero también se presentaba co-destrucción en la medida que dichos elementos fueron incongruentes para ellos.

Llevó a cabo ejercicios con estudiantes de pregrado para cocrear planes de estudio. Iniciar la co-

Servicios

-Educación

(Bovill, Superior creación temprana puede ayudar a los estudiantes a desarrollar

Argumenta que es posible que los estudiantes no puedan contribuir de manera significativa al contenido del currículo el primer día, pero incluso pequeños aumentos de elección en las primeras etapas de las experiencias de aprendizaje negociación y la comprensión universitario pueden comunicar el mensaje de que metacognitiva del proceso de la participación del estudiante en la enseñanza y el aprendizaje para el final de su aprendizaje es bienvenida. carrera. 
ASOCIACIONES CRIITICAS

Lo que hasta hoy se ha denominado co-creación de valor, es una evolución que proporciona a las empresas un impulso estratégico para reconsiderar los roles de sus clientes en las prácticas de marketing actuales (Prahalad \& Ramaswamy, 2004a), y que, ubicada en una época de base financiera, es el propósito principal y proceso central de intercambio económico (Vargo, Maglio, \& Akaka, 2008).

Sin embargo, de acuerdo a (Greer, 2015), el comportamiento del consumidor disfuncional puede obstaculizar la co-creación de valor, por ejemplo con la agresión física y el abuso verbal, repercutiendo esto en dos formas de mala conducta del consumidor que pueden variar, aumentar o coincidir durante los encuentros del servicio, ya sea por una participación insuficiente o por una sobreparticipación. Esto puede traer implicaciones negativas en la administración de las organizaciones, pues los resultados positivos no se garantizan siguiendo un enfoque de co-creación debido a los diversos riesgos asociados a esta "técnica de gestión" que también es evaluada desde perspectivas culturales, sociales y económicas (Zwick, Bonsu, \& Darmody, 2008).

Asimismo, el aumento de la participación de los clientes (sobreparticipación) reduce el control que la empresa tiene sobre el resultado del proceso de co-creación de valor, lo que introduce un mayor nivel de incertidumbre y riesgo (Zhuang, 2010). De otro lado, los clientes podrían no mostrar los conocimientos y habilidades necesarios para aceptar con éxito la propuesta de valor de la organización, siendo posible que no se genere la co-creación (Terblanche, 2014).

Al respecto (Chan, Yim, \& Lam, 2010) reconocen que, efectivamente, la co-creación genera beneficios económicos para los clientes, fortaleciendo el vínculo emocional de éstos con la empresa, pero sostienen que al mismo tiempo aumenta el estrés laboral de los empleados, causando dificultades en su satisfacción laboral. Adicionalmente, explican que el valor creado por la colaboración conjunta entre ambos agentes depende de sus valores culturales, por lo cual debe existir una "alineación" entre clientes y empleados en función de dichos valores asegurando el éxito en la co-creación. En otras palabras, sostienen estos autores que, cuando los elementos de las prácticas son incongruentes, y las partes no pueden ponerse de acuerdo en su interacción sobre procedimientos y demás, se produce una co-destrucción de valor.

Del mismo modo, coinciden las apreciaciones de Plé \& Cáceres, (2010) al afirmar que la co-creación de valor ocurre cuando dos sistemas de servicio presentan expectativas congruentes sobre la forma en que se deben usar los recursos disponibles a lo largo de sus interacciones, por tanto, el uso inapropiado o inesperado de los recursos disponibles en una interacción, dará como resultado la destrucción conjunta de valor -para al menos una de las partes intervinientes-. Dichos autores sugieren que la co-destrucción de valor se puede definir como “...un proceso internacional entre sistemas de servicio que resultan en una disminución de bienestar, al menos para uno de los sistemas”. Durante este proceso los sistemas de servicio interactúan, ya sea directamente (persona a persona) o indirectamente (con un producto, por ejemplo) a través de la integración y aplicación de los recursos.

Como respuesta a las críticas y controversias generadas, Vargo \& Akaka, (2009) discutieron la lógica DS como una base para la ciencia del servicio de acuerdo a sus premisas fundantes con el fin de "aclarar" interpretaciones erróneas de otros autores con respecto al papel que ejerce el servicio en el ámbito económico y la naturaleza, por ende, de la co-creación de valor. Sostienen en su trabajo, que la ciencia del servicio es una disciplina emergente que se ocupa de la evolución, la interacción y la creación recíproca de valor entre los sistemas de servicio (Spohrer \& Maglio, 2008), (Chen, Spohrer, \& Lelescu, 2008). Por tanto, argumentan que la lógica DS (Vargo \& Lusch, 2004, 2008) es una alternativa al paradigma tradicional (dominancia de los bienes), para entender el intercambio económico y la creación de valor como las bases del servicio, lo cual -a su juicio- ha sido malinterpretado o tergiversado. 
Un análisis crítico a lo anteriormente mencionado, por parte de Grönroos (2011), afirma que algunas de las premisas fundamentales de la lógica SD no respaldan a cabalidad la comprensión de la co-creación de valor de manera substancial para el desarrollo teórico y la toma de decisiones en el marketing, pues argumenta que no existe profundidad en el concepto de interacción y que, por esto, la co-creación de valor se convierte fácilmente en un algo sin sustancia, pues sigue siendo imprecisa su naturaleza y su contenido. Dentro de dicho análisis logra observar que no solo los clientes son co-creadores de valor, sino que -bajo ciertas circunstancias- el proveedor del servicio posee oportunidades para crear valor conjuntamente con ellos. Incluso, el autor ha seguido ratificando sus contradicciones sobre la lógica SD mencionando que se basa en una visión metafórica de co-creación de valor, encarcelándose a sí misma (Grönroos \& Gummerus, 2014), pues ésta será libre y exitosa, siempre y cuando intervengan en el proceso las participaciones de la empresa, los clientes y otros actores, permitiendo reinventar significativamente el marketing desde una perspectiva de servicio.

De otro lado, son Frow \& Payne (2011) quienes hacen una crítica a la lógica SD porque, desde su perspectiva, las partes interesadas (stakeholders) han recibido muy poca atención. Debido a ello, plantean una nueva percepción de la creación de valor dentro de una red de valor, considerando así una visión más amplia que permite unir el concepto de stakeholder con la co-creación de valor. De esa manera, desarrollan un proceso de cinco pasos para identificar quiénes son las partes interesadas clave para la organización, con el propósito de crear colaborativamente con ellas, propuestas de valor dentro de un sistema de comercialización, proporcionando mecanismos de alineación del valor dentro de un sistema de marketing. Esto, sin duda, brinda oportunidades estratégicas a los gerentes para asegurar y estabilizar las relaciones dentro de la red de valor de sus organizaciones.

En esta misma línea, en años más recientes, Laamanen \& Skalen (2015), esbozaron un marco conceptual para la co-creación de valor centrándose en una acción colectiva donde incluyen -no solo al cliente- sino a múltiples actores anidados que enriquecen las interacciones, las prácticas y los resultados de este proceso del marketing. Y (Dong \& Sivakumar, 2017), van más allá de estos hechos, pues sugieren usar el término "participación del cliente" (PC) en reemplazo de otros conceptos como co-producción y co-creación, contribuyendo a reducir confusiones; de la misma manera, utilizar de manera diferente los conceptos de PC, compromiso del cliente e innovación del cliente, así se relacionen entre ellos; sin embargo, sobre esta base conceptual mejorada propuesta por los autores, dejan abiertas las discusiones e implicaciones gerenciales y las futuras orientaciones investigativas en este campo.

\section{DISCUSIÓN}

El término "co-tendencias" se propone por la autora para indicar una correspondencia con la co-creación de valor, pues si ésta alude a la creación de valor de manera conjunta, el concepto co-tendencia indica la forma colaborativa en la que se propendería o se proyectarían nuevas situaciones relacionadas con la co-creación de valor. Siendo así, es primordial comprender que desde comienzos del nuevo milenio ya Prahalad \& Ramaswamy (2003, 2004a) mencionaban la "alquimia de la innovación" dentro de los procesos comerciales que reclamaban un papel mayormente activo de los consumidores en la sociedad.

Introducida la segunda década son Lusch \& Webster Jr., (2011), quienes argumentan lo fundamental que es para las empresas operar como si el marketing ya no fuera simplemente una función comercial separada, sino una responsabilidad general de la gestión dentro de una red muy amplia de partes interesadas, las cuales deben unificarse con el cliente y con la organización. El foco está en las interdependencias dadas entre la configuración de recursos en un sistema de servicio y los esquemas que dan forma a los clientes y otros actores, a medida que se integran dichos recursos y co-crean valor (Edvardsson \& Tronvoll, 2013). 
Una marcada co-tendencia se explica desde lo expuesto por Jaakkola \& Alexander (2014) al resaltar la difuminación de fronteras entre las empresas y los clientes, pues los comportamientos de éstos y su alto nivel de involucramiento en el sistema de servicio, hace que vayan más allá de una simple transacción o relación comercial. Por ello, estos autores marcan el primer intento para conceptualizar el comportamiento del consumidor desde ese nivel de compromiso o involucramiento en la co-creación de valor, proporcionando así, una luz sobre el rol fundamental que juegan las proposiciones de valor en los sistemas de servicio (Chandler \& Lusch, 2015).

Finalmente, dentro de las investigaciones más recientes se encuentra una co-tendencia en la cual se hace un llamado a la reflexión en cuanto a que la mirada sobre ese comportamiento del consumidor, desde su nivel de compromiso o involucramiento, sea de 360 grados (Finsterwalder, 2018), pues se han descuidado las funciones de otros stakeholders, como los empleados por ejemplo, quienes pueden desempeñar un papel trascendental en el proceso de co-creación de valor. Por tanto, en un sistema de servicio invitan estos autores a centrarse en todos los actores que tienen intención de participar, en los que ya participan de manera activa, e incluso, en los que ya están desconectados del proceso de co-creación de valor.

\section{CONCLUSIONES}

1) Conceptualmente se infiere que la co-creación de valor es un término que no se ha definido aún de manera precisa, pues son muchas las perspectivas desde las cuales se ha querido abordar. Si bien es cierto que, la lógica del SD ha sido la teoría bajo la cual se ha introducido y crecido este concepto, se puede afirmar que todavía no llega a una etapa de madurez donde exista una amplia y recorrida trayectoria en sus aplicaciones y sus metodologías.

2) Aunque fue en la primera década del S. XXI que la co-creación de valor tuvo su apogeo y se comenzó a elevar el conocimiento en este importante campo del marketing, han sido los albores de esta segunda década del nuevo milenio, los que han ocasionado una gran avalancha de conflictos y controversias, pues van en aumento los autores que perciben un "encarcelamiento" en la co-creación de valor, pues diera la impresión de seguir maniatada a la teoría fundamente de la lógica SD, donde la piedra angular ha sido el servicio, pero limitado en sus alcances y sus propósitos. A este respecto, es fundamental conocer aún más las críticas que han desencadenado distintos autores, con el único fin de reestablecer para la co-creación de valor unos mecanismos estratégicos que permitan, por un lado, llegar a un significado que sea común y conocido para la comunidad del campo de conocimiento, y por otro, lograr -quizás- que pueda llegar a convertirse en una herramienta fundamental para la gestión de las organizaciones, pues no se puede desconocer que es el marketing el que trabaja más cerca al cliente y a los stakeholders.

3) Mayores apuestas en la comprensión de las co-tendencias y las problemáticas asociadas a la co-creación de valor serán siempre puerta de entrada para ahondar en la contribución que este proceso del marketing hace a la gestión de las organizaciones, pues es un fenómeno interesante y de actualidad, incluido en las últimas tendencias del marketing consideradas en los tópicos prioritarios de investigación expuestos por el Instituto de Ciencias del Marketing (MSI), la Asociación Americana de Marketing (AMA) y los temas de discusión recomendados en las últimas publicaciones de los Journals reconocidos en la disciplina, tal y como se evidencia en esta RSL.

4) Para la Administración y las organizaciones en sí, la co-creación de valor es un proceso complejo en el que deben participar varios actores, no solamente la empresa como oferente de productos y servicios con sus clientes, sino también aquellos que guardan estrechas relaciones e interacciones como: los proveedores, distribuidores, competidores, colaboradores y socios. 
Y aunque son variados los escritos que se han publicado sobre co-creación de valor mostrando sus orígenes, beneficios, implicaciones, modelos, ejemplos y articulaciones con otros conceptos o campos de conocimiento, sigue siendo necesario encontrar nuevas herramientas o mecanismos para su significación y utilización, llevando esto a descubrir nuevos enfoques en la aplicación del marketing para la gestión de las organizaciones.

\section{REFERENCIAS}

Aarikka-Stenroos, L., \& Jaakkola, E. (2012). Value co-creation in knowledge intensive business services: A dyadic perspective on the joint problem solving process. Industrial Marketing Management, 41(1), 15-26. https://doi. org/10.1016/j.indmarman.2011.11.008

Akaka, M. A., Corsaro, D., Kelleher, C., Maglio, P. P., Seo, Y., Lusch, R. F., \& Vargo, S. L. (2014). The role of symbols in value co-creation. Marketing Theory, 14(3), 311-326. https://doi. org/10.1177/1470593114534344

Akesson, M., Edvardsson, B., Tronvoll, B., Åkesson, M., Edvardsson, B., \& ... (2014). Customer experience from a self-service system perspective. Journal of service management, 25(5, SI), 677-698. https://doi.org/10.1108/JOSM01-2013-0016

Andajani, E. (2015). Understanding Customer Experience. Management in Retailing. Procedia- Social and Behavioral Sciences, 211 (November 2015), 629-633. https://doi.org/10.1016/j.sbspro.2015.11.082

Bolderston, Amanda. (2008). Writing an effective Literature Review. Journal of Medical Imaging and Radiation Sciences, Volume 39 (2), 86 - 92. https://doi. org/10.1016/j.jmir.2008.04.009

Bovill, C. (2015). Students and staff co-creating curricula: an example of good practice in higher education? In E. G. P. Limited (Ed.), The Student Engagement Handbook: Practice in $\mathrm{Hi}-$ gher Education. Emerald Group Publishing Ltd.

Chan, K. W., Yim, C. K., \& Lam, S. S. $\mathrm{K}$. (2010). Is customer participation in value creation a double-edged sword?
Evidence from professional financial services across cultures. Journal of Marketing. Retrieved from http://journals. ama.org/doi/abs/10.1509/jmkg.74.3.48

Chandler, J. D., \& Lusch, R. F. (2015). Service Systems: A Broadened Framework and Research Agenda on Value Propositions, Engagement, and Service Experience. Journal of Service Research, 18(1), 6-22. https://doi. org/10.1177/1094670514537709

Chen, Y., Spohrer, J., \& Lelescu, A. (2008). Three factors to sustainable service system excellence: A case study of service systems. Services Computing, 2008 .... Retrieved from http://ieeexplore.ieee. org/abstract/document/4578516/

Cova, B., \& Salle, R. (2008). Marketing solutions in accordance with the S-D logic: Co-creating value with customer network actors. Industrial Marketing Management, 37(3), 270-277. https://doi.org/10.1016/j.indmarman.2007.07.005

Dong, B., \& Sivakumar, K. (2017). Customer participation in services: domain, scope, and boundaries. Journal of the Academy of Marketing Science, 45(6, SI), 944-965. https://doi.org/10.1007/ S11747-017-0524-y

Echeverri, P., Skålén, P., \& Skálen, P. (2011). Co-creation and co-destruction: A practice-theory based study of interactive value formation. Marketing Theory, 11(3), 351-373. https://doi. org/10.1177/1470593111408181

Edvardsson, B., Skålén, P., \& Tronvoll, B. (2012). Service systems as a foundation for resource integration and value co-creation. Review of Marketing Research, 9, 79-126. https://doi.org/10.1108/S15486435(2012)0000009008

Edvardsson, B., \& Tronvoll, B. (2013). A new conceptualization of service innovation grounded in S-D logic and service systems. International Journal of Quality and Service Sciences, 5(1), 19-31. https://doi.org/10.1108/17566691311316220

Finsterwalder, J. (2018). A 360-degree view of actor engagement in service co-creation. Journal of Retailing and Consumer Services, 40, 276-278. https://doi. org/10.1016/j.jretconser.2016.08.005

Frow, P., \& Payne, A. (2011). A stakeholder perspective of the value proposition concept. European Journal of Mar- 
keting, 45(1/2), 223-240. https://doi. org/10.1108/03090561111095676

Frow, P., Nenonen, S., Payne, A., \& ... (2015). Managing co-creation design: A strategic approach to innovation. British Journal of Management, 26, 463-483. https://doi.org/10.1111/1467-8551.12087

Greer, D. A. (2015). Defective co-creation Developing a typology of consumer dysfunction in professional services. European journal of marketing, 49(1-2), 238-261. https://doi.org/10.1108/EJM07-2012-0411

Grönroos, C. (2011). Value co-creation in service logic: A critical analysis. Marketing Theory, $11(3$, SI), 279-301. https://doi.org/10.1177/1470593111408177

Grönroos, C. (2015). From Marketing Mix to Relationship Marketing: towards a paradigm shift in marketing. Management Decision, 32(2), 4-20.

Grönroos, C., \& Gummerus, J. (2014). The service revolution and its marketing implications: service logic vs service-dominant logic. Managing Service Quality, 24(3), 206-229. https://doi. org/10.1108/MSQ-03-2014-0042

Grönroos, C., \& Ravald, A. (2011). Service as business logic: implications for value creation and marketing. Journal of Service Management, 22 (January 2014), 5-22. https://doi. org/10.1108/09564231111106893

Gummesson, E. (2012). The three service marketing paradigms: which one are you guided by? Stockholm: FrancoAngeli. https://doi.org/10.3280/MC2012001001

Hilton, T., Hughes, T., \& Chalcraft, D. (2012). Service co-creation and value realisation. Journal of Marketing $\mathrm{Ma}-$ nagement, 28(13-14), 1504-1519. https:// doi.org/10.1080/0267257X.2012.736874 Jaakkola, E., \& Alexander, M. (2014). The Role of Customer Engagement Behavior in Value Co-Creation: A Service System Perspective. Journal of Service Research, 17(3), 247-261. https://doi. org/10.1177/1094670514529187

Jadika, K., Khoo, C., \& Na, J. (2011). Literature Review Writing: A Study of Information Selection from Cited Papers. In Asia Pacific Conference Library \& Information Education \& Practice (pp. 467-477).

Laamanen, M., \& Skalen, P. (2015). Collective-conflictual value co-creation: A strategic action field approach. Marketing Theory, 15(3), 381-40o. https://doi. org/10.1177/1470593114564905

Lusch, R. F., \& Webster Jr., F. E. (2011). A Stakeholder-Unifying, cocreation philosophy for marketing. Journal of Macromarketing, 31(2), 129-134. https:// doi.org/10.1177/0276146710397369

McColl-Kennedy, J. R., Vargo, S. L., Dagger, T. S., Sweeney, J. C., \& van Kasteren, Y. (2012). Health Care Customer Value Cocreation Practice Styles. Journal of Service Research, 15(4), 370-389. https://doi.org/10.1177/1094670512442806

Meyer, C., \& Schwager, A. (2007). Understanding Customer Experience. Harvard Business Review, 85(2), 116-124. https:// doi.org/10.1108/00242539410067746

Meynhardt, T., Chandler, J. D., \& Strathoff, P. (2016). Systemic principles of value co-creation: Synergetics of value and service ecosystems. Journal of Business Research, 69(8), 2981-2989. https://doi. org/10.1016/j.jbusres.2016.02.031

Murphy, C. M. (2012). Writing an Effective Review Article. Journal of Medical Toxicology, 8(2), 89-90. https://doi. org/10.1007/s13181-012-0234-2

Pautasso, M. (2013). Ten Simple Rules for Writing a Literature Review. PLoS Computational Biology, 9(7), 7-10. https:// doi.org/10.1371/journal.pcbi.1003149

Payne, A., Storbacka, K., Frow, P., \& Knox, S. (2009). Co-creating brands: Diagnosing and designing the relationship experience. Journal of Business Research, 62(3), 379-389. https:// doi.org/10.1016/j.jbusres.2008.05.013

Pine II, B. . J. , \& Gilmore, J. H. . (1998). Welcome to the experience economy. Harvard Business Review, 4 (August), 97-105. Retrieved from https://www.researchgate.net/publication/299292969_ The_Experience_Economy

Plé, L. (2016). Studying customers' resource integration by service employees in interactional value co-creation. Journal of Services Marketing, 30(2), 152-164. https://doi.org/10.1108/JSM-02-20150065

Plé, L., \& Cáceres, R. C. (2010). Not always co-creation: introducing interactional co-destruction of value in service-dominant logic. Journal of Services Marketing, (June 2016). https://doi. org/10.1108/08876041011072546

Polese, F., Mele, C., \& Gummesson, E. 
(2017). Value co-creation as a complex adaptive process. Journal of Service Theory and Practice, 27(5), 926-929. https://doi.org/10.1108/JSTP-07-2017-0111

Prahalad, C. K. , \& Ramaswamy, V. (2000). Co-opting customer competence. Harvard Business Review. USA.

Prahalad, C. K., \& Ramaswamy, V. (2003). The new frontier of experience innovation. MIT Sloan Management Review, 44(4), 12-18. Retrieved from https://sloanreview.mit.edu/article/ the-new-frontier-of-experience-innovation/

Prahalad, C. K., \& Ramaswamy, V. (2004a). Co-creating unique value with customers. Strategy \& Leadership, 32(3), 4-9. Retrieved from http://www. cs.cmu.edu/ jhm/Readings/Co-creating unique value with customers.pdf

Prahalad, C. K., \& Ramaswamy, V. (2004b). Co-creation experiences: the next practice in value creation. Journal of Interactive Marketing, 18(3), 5-14. https://doi.org/10.1002/dir.20015

Randolph, Justus. (2009). A Guide to Writing the Dissertation Literature $\mathrm{Re}$ view. Practical Assessment, Research \& Evaluation, 14(13).

Ranjan, K. R., \& Read, S. (2014). Value co-creation: concept and measurement. Journal of the Academy of Marketing Science, 44(3), 290-315. https:// doi.org/10.1007/s11747-014-0397-2

Saarijärvi, H. (2012). The mechanisms of value co-creation. Journal of Strategic Marketing, 20(5), 381-391. https://doi. org/10.1080/og65254X.2012.671339

Scimago Journal and Country Rank. Available at: https://www.scimagojr.com/

Spohrer, J., \& Maglio, P. P. (2008). The emergence of service science: Toward systematic service innovations to accelerate co-creation of value. Production and Operations Management, 17(3), 238-246. https://doi.org/10.3401/ poms.1080.0027

Suter, G. W. (2013). Review papers are important and worth writing. Environmental Toxicology and Chemistry, 32(9), 1929-1930. https://doi. org/10.1002/etc. 2316

Terblanche, N. S. (2014). Some theoretical perspectives of co-creation and co-production of value by customers. Acta Commercii, 1-8. https://doi. org/10.4102/ac.v14i2.237

Vargo, S. L., \& Akaka, M. A. (2009). Service-dominant logic as a foundation for service science: clarifications. Service Science, 1(1), 32-41. https://doi. org/10.1287/serv.1.1.32

Vargo, S. L., \& Lusch, R. F. (2004). Service dominant logic. Journal of Marketing, 1-20.

Vargo, S. L., \& Lusch, R. F. (2008). Service-dominant logic: continuing the evolution. Journal of the Academy of Marketing Science, 36, 1-10. https:// doi.org/10.1007/s11747-007-0069-6

Vargo, S. L., \& Lusch, R. F. (2017). Service-dominant logic 2025. International Journal of Research in Marketing, 34(1), 46-67. https://doi.org/10.1016/j. ijresmar.2016.11.001

Vargo, S. L., Maglio, P. P., \& Akaka, M. A. (2008). On value and value co-creation: A service systems and service logic perspective. European Management Journal, 26, 145-152. https://doi. org/10.1016/j.emj.2008.04.003

Vega-Vazquez, M., Revilla-Camacho, M. Á., \& Cossío-Silva, F. J. (2013). The value co-creation process as a determinant of customer satisfaction. Management Decision, 51, 1945-1953. https://doi. org/10.1108/MD-04-2013-0227

Verhoef, P. C., Lemon, K. N., Parasuraman, A., Roggeveen, A., Tsiros, M., \& Schlesinger, L. A. (2009). Customer experience creation: determinants, dynamics and management strategies. Journal of Retailing, 85(1), 31-41. https:// doi.org/10.1016/j.jretai.2008.11.001

Zhuang, W. (2010). Balancing customer and marketing inputs to maximize the value experience. PhD Thesis, Department of Marketing and Analysis, Louisiana Tech University.

Zwick, D., Bonsu, S. K., \& Darmody, A. (2008). Putting consumers to work: "Co-creation" and new marketing govern-mentality. Journal of Consumer Culture, 8(2), 163-196. https://doi. org/10.1177/1469540508090089 